\title{
Modelling occupants' personal characteristics for thermal comfort prediction
}

\author{
Frédéric Haldi • Darren Robinson
}

Received: 9 July 2010 /Revised: 14 October 2010 /Accepted: 17 October 2010 /Published online: 25 February 2011

(C) ISB 2011

\begin{abstract}
Based on results from a field survey campaign conducted in Switzerand, we show that occupants' variations in clothing choices, which are relatively unconstrained, are best described by the daily mean outdoor temperature and that major clothing adjustments occur rarely during the day. We then develop an ordinal logistic model of the probability distribution of discretised clothing levels, which results in a concise and informative expression of occupants' clothing choices. Results from both cross-validation and independent verification suggest that this model formulation may be used with confidence. Furthermore, the form of the model is readily generalisable, given the requisite calibration data, to environments where dress codes are more specific. We also observe that, for these building occupants, the prevailing metabolic activity levels are mostly constant for the whole range of surveyed environmental conditions, as their activities are relatively constrained by the tasks in hand. Occupants may compensate for this constraint, however, through the consumption of cold and hot drinks, with corresponding impacts on metabolic heat production. Indeed, cold drink consumption was found to be highly correlated with indoor thermal conditions, whilst hot drink consumption is best described by a seasonal variable. These variables can be used for predictive purposes using binary logistic models.
\end{abstract}

Keywords Clothing · Metabolic activity · Drinks ·

Adaptive actions $\cdot$ Behavioural modelling

F. Haldi $(\bowtie) \cdot$ D. Robinson

Solar Energy and Building Physics Laboratory (LESO-PB),

Ecole Polytechnique Fédérale de Lausanne (EPFL),

CH-1015, Lausanne, Switzerland

e-mail: frederic.haldi@a3.epfl.ch

\section{Introduction}

The deterministic features of building simulation programs are now considered relatively mature. But their ability to emulate reality is undermined by a poor representation of non-deterministic variables, particularly relating to occupants' presence, their interactions with environmental controls and adaptations of their personal characteristics (such as clothing and metabolic activity); which impact buildings' heat gains and occupants' environmental comfort.

The prediction of clothing level, metabolic activity and cold or hot drinks consumption is of indirect relevance to building performance simulation. Unlike windows and blinds, these variables have only a marginal influence on the heat gains of a building, due to occupants' metabolic heat gains. However, their impact on occupants' comfort is significant and this may influence occupants' actions to adapt the building envelope. This article presents a new approach for the prediction of occupants' choices of clothing level, together with occupants' probable metabolic activity and their consumption of cold and hot drinks. For each of these topics we begin with a short review of previously published research, but first we introduce the field survey and the experimental design from which our findings are developed.

\section{Materials and methods}

The Solar Energy and Building Physics Laboratory (LESOPB) experimental building (Fig. 1, left), located in the suburbs of Lausanne, Switzerland $\left(46^{\circ} 31^{\prime} 17^{\prime \prime} \mathrm{N}, 6^{\circ} 34^{\prime} 02^{\prime \prime} \mathrm{E}\right.$, alt. $396 \mathrm{~m}$.) was built in 1982 and renovated in 1999. Apart from service rooms, it hosts on three floors 14 south-facing offices, which have an area of $15.7 \mathrm{~m}^{2}$ and a height of 
Fig. 1 View of the south façade of the LESO building (left) and typical cellular office (right)
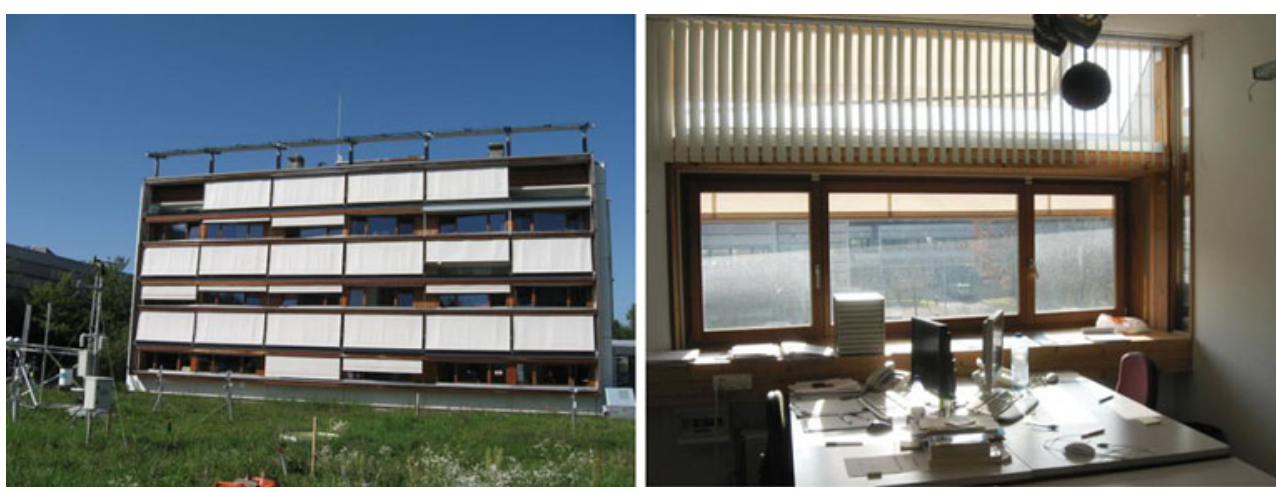

$2.8 \mathrm{~m}$, equipped with anidolic systems that improve the distribution of daylight. A typical office is shown in Fig. 1 (right). A detailed description of the building with an exhaustive analysis of the building's energy flows is provided by Altherr and Gay (2002).

In every south-facing office, occupants have the possibility to tilt or open up to any angle each of the two windows (height $0.9 \mathrm{~m}$, width $0.7 \mathrm{~m}$ ). They also have the possibility to control two external blinds (width $3.50 \mathrm{~m}$ ): a lower blind potentially covering the totality of the vision window (height $1.00-1.85 \mathrm{~m}$ ) and an upper blind covering the anidolic system (height $2.10-2.70 \mathrm{~m}$ ). Electrical lighting may be dimmed to any desired level with a switch located next to each office door.

Occupants of this building include senior researchers, research assistants, technical staff and secretaries. They mainly carry out office-related work and all use a computer. There is no particular prevailing dress code. During the surveyed period, between five and eight offices were occupied by two persons, while between six and nine offices accommodated single occupants who were able to act on the two windows.

All 14 south-facing cellular offices of this building are equipped with sensors whose real-time measurements are archived by a centralised EIB data acquisition system. Occupants' presence (measured by infrared sensors in every office), and local indoor $\left(\theta_{\text {in }}\right)$ and outdoor $\left(\theta_{\text {out }}\right)$ air temperature (measured by Pt-100 resistance thermometers in the offices and on the roof) have been continuously recorded; likewise, a range of other variables.

An electronic questionnaire (Fig. 2) was activated on the computers of all participating occupants on a rotation basis, first from 13 June to 27 September 2006 and then from 13 February 2008 to 8 September 2009. Where possible, each occupant was surveyed for at least three separate periods of 3 months in winter, in summer and during an inter-seasonal period. The questionnaire typically appeared four times a day, twice in the morning and in the afternoon, at intervals of between 2 and $3 \mathrm{~h}$ (defined in agreement with each occupant).
At every prompt, occupants were asked to provide the following information:

Current clothing level $\left(I_{c l}\right)$ The surveyed occupants could choose from amongst eight possibilities proposed in a dropdown list (Table 1, upper), from which typical clothing insulation values ranging from 0.3 to 0.95 (clo) could be deduced from the ISO 7730 standard.

Activity level during the preceding 15 minutes (M) Six possibilities were offered (Table 1, lower), also based on the ISO 7730 standard. Respondents could also tick a "no change" box, following from the first prompt of the day.

Thermal, visual and olfactory sensation $\left(S_{t h}, S_{v i s}, S_{\text {olf }}\right)$ An approximate French translation of the standard seven-point ASHRAE scale for thermal sensation (itself used in the ISO 7730 standard) was proposed. A similar seven-point bidirectional scale was chosen for visual sensation, whereas a seven-point unidirectional scale was used for olfactory sensation. Findings based on these scales are presented by Haldi and Robinson (2010b).

Other activities during the preceding hour These include the intake of hot and cold drinks, meals, and additions or removals of clothing items.

The questionnaire produced 3,924 valid answers obtained from 17 occupants ( 8 male, 9 female), Table 2 provides a statistical summary of the variables of interest.

\section{Clothing}

State of the art

The issue of clothing can be seen from different perspectives. As pointed out by Morgan and De Dear (2003), clothing has an ergonomic function; but it is also affected by cultural and social contexts, by personality and by corporate identity, while 
Fig. 2 Electronic questionnaire: main survey window

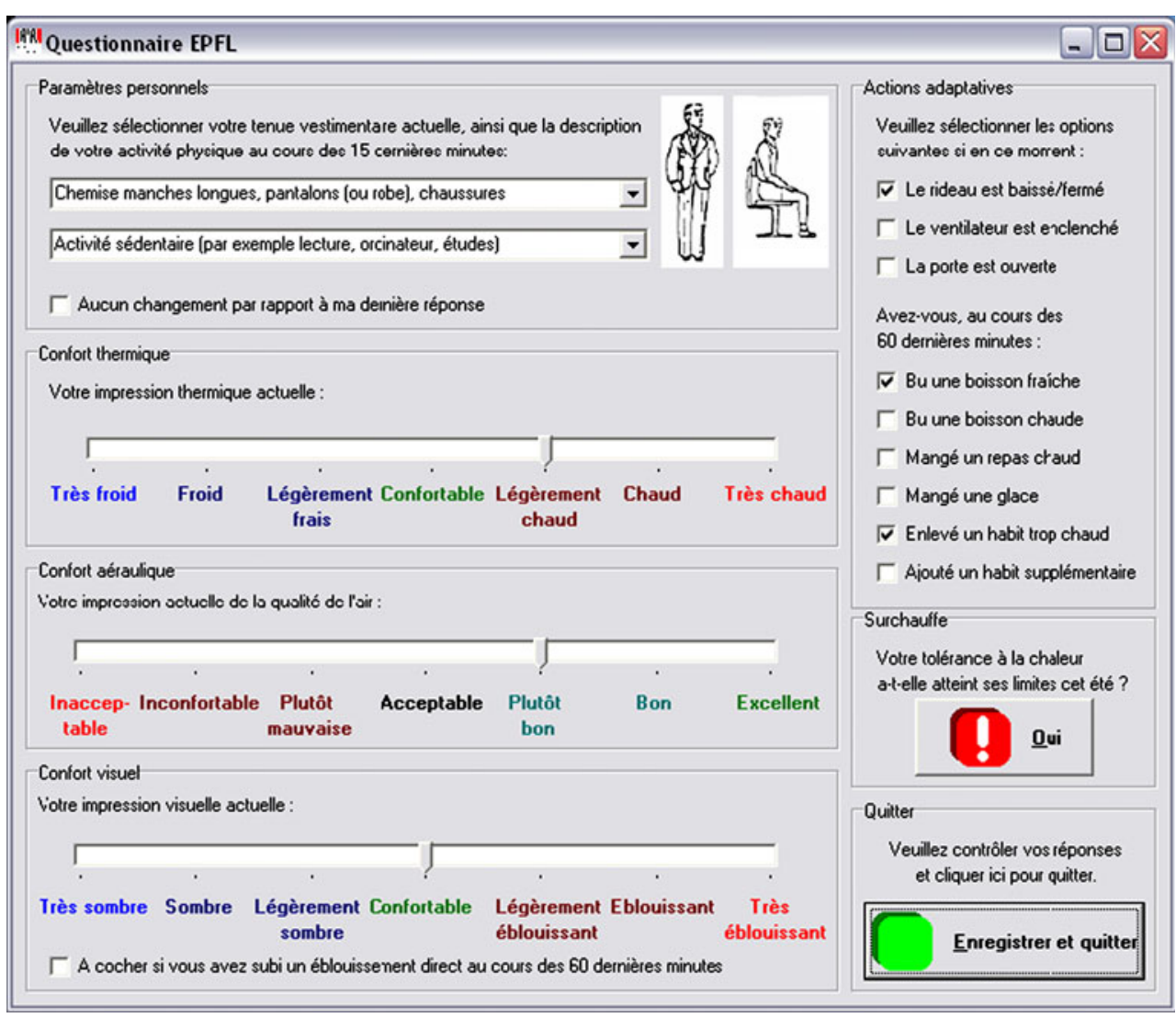

Table 1 Choices available in the electronic questionnaire for clothing and activity, with corresponding values from ISO-7730 Standard

\begin{tabular}{lll}
\hline Clothing ensemble (English translation) $\quad$ Clothing ensemble (original text) & Insulation \\
\cline { 2 - 2 } & $\left(\mathrm{m}^{2} \mathrm{~K} / \mathrm{W}\right)$ \\
\hline
\end{tabular}

Jacket, shirt with long sleeves, trousers/dress, tie, shoes

Jacket, open neck shirt, trousers/dress, shoes

Shirt with long sleeves, trousers/dress, tie, shoes

Shirt with long sleeves, trousers/dress, shoes

Sweater, shirt, trousers, shoes

Shirt with short sleeves, trousers, shoes

Shirt with short sleeves, trousers, sandals

Shirt with short sleeves, short/skirt, shoes

Shirt with short sleeves, short/skirt, sandals
Veston, chemise manches longues, pantalons/robe, cravate, chaussures

Veston, chemise à col ouvert, pantalons/robe, chaussures

Chemise manches longues, pantalons ou robe, cravate, chaussures

Chemise manches longues, pantalons/robe, chaussures

Pullover, chemise, pantalons, chaussures

Chemise manches courtes, pantalons/robe, chaussures

Chemise manches courtes, pantalons/robe, sandales

Chemise manches courtes, shorts/jupe, chaussures

Chemise manches courtes, shorts/jupe, sandales
0.147

0.140

0.124

0.116

0.109

0.093

0.080

0.062

0.047

Metabolic rates

Activity (original text)

$\left(\mathrm{W} / \mathrm{m}^{2}\right) \quad(\mathrm{met})$

Seated, relaxed

Sedentary activity

Standing, light activity

Standing, medium activity

Walking

Cycling, running
Assis, inactif

Activité sédentaire

Activité légère, debout

Travail debout

Marche

Cyclisme, course

$\begin{array}{ll}58 & 1.0 \\ 70 & 1.2 \\ 93 & 1.6 \\ 116 & 2.0 \\ 140 & 2.4 \\ 232 & 4.0\end{array}$


Table 2 Statistical summary of the questionnaire items: occupant reference number, gender and class of age, number of completed entries, mean reported clothing level and metabolic activity, proportion of answers reporting drink intake and clothing adjustments

\begin{tabular}{|c|c|c|c|c|c|c|c|c|c|}
\hline Ref. & Gender & Age class & No. of entries & $\mathrm{I}_{\mathrm{cl}}(\mathrm{clo})$ & M (met) & Cold drinks & Hot drinks & Clothing additions & Clothing removals \\
\hline 1 & M & $20-30$ & 51 & 0.72 & 1.28 & 0.67 & 0.06 & $<0.001$ & 0.235 \\
\hline 2 & $\mathrm{~F}$ & $20-30$ & 186 & 0.66 & 1.38 & 0.09 & 0.01 & $<0.001$ & $<0.001$ \\
\hline 3 & $\mathrm{~F}$ & $20-30$ & 120 & 0.56 & 1.60 & 0.07 & 0.01 & $<0.001$ & $<0.001$ \\
\hline 4 & $\mathrm{~F}$ & $50-65$ & 441 & 0.74 & 1.34 & 0.16 & 0.37 & $<0.001$ & 0.056 \\
\hline 5 & M & $50-65$ & 143 & 0.61 & 1.22 & 0.01 & 0.02 & $<0.001$ & 0.010 \\
\hline 6 & M & $30-40$ & 263 & 0.61 & 1.26 & 0.73 & 0.12 & $<0.001$ & 0.013 \\
\hline 7 & M & $20-30$ & 383 & 0.68 & 1.23 & 0.10 & 0.17 & $<0.001$ & $<0.001$ \\
\hline 8 & M & $20-30$ & 415 & 0.61 & 1.30 & 0.14 & 0.24 & 0.005 & 0.007 \\
\hline 9 & M & $20-30$ & 38 & 0.74 & 1.20 & 0.13 & 0.03 & $<0.001$ & 0.026 \\
\hline 10 & $\mathrm{~F}$ & $20-30$ & 138 & 0.66 & 1.58 & 0.12 & 0.01 & $<0.001$ & $<0.001$ \\
\hline 11 & $\mathrm{~F}$ & $30-40$ & 312 & 0.66 & 1.32 & 0.02 & 0.06 & $<0.001$ & $<0.001$ \\
\hline 12 & $\mathrm{~F}$ & $20-30$ & 409 & 0.68 & 1.28 & 0.18 & 0.16 & 0.007 & 0.011 \\
\hline 13 & M & $50-65$ & 372 & 0.37 & 1.28 & 0.60 & 0.05 & 0.005 & 0.072 \\
\hline 14 & $\mathrm{~F}$ & $30-40$ & 35 & 0.86 & 1.21 & 0.03 & 0.09 & $<0.001$ & $<0.001$ \\
\hline 15 & M & $50-65$ & 272 & 0.65 & 1.49 & 0.01 & 0.04 & $<0.001$ & $<0.001$ \\
\hline 16 & $\mathrm{~F}$ & $50-65$ & 115 & 0.67 & 1.22 & 0.03 & 0.03 & $<0.001$ & $<0.001$ \\
\hline 17 & $\mathrm{~F}$ & $50-65$ & 231 & 0.87 & 1.20 & 0.04 & 0.23 & $<0.001$ & 0.004 \\
\hline Total & & & 3,924 & 0.65 & 1.32 & 0.19 & 0.14 & 0.002 & 0.019 \\
\hline
\end{tabular}

its primary function is to serve as a simple layer of thermal insulation. Although in this work we are particularly interested in the latter function, it is important to bear in mind other factors which motivate our clothing choices.

Clothing has an important impact on the human heat balance. As such, the reliable estimation of clothing level is important for the estimation of thermal satisfaction, e.g. using the ISO-7730 standard (ISO 2005), where clothing level is a key variable. It is, however, difficult to perform precise estimates from field surveys without invasive methods. Methods thus far employed for the estimation of clothing level are discussed by Olesen (1985).

A first field study on clothing behaviour was carried out by Humphreys $(1972,1973)$ on secondary school children. It was observed that the proportion of children stripped to the minimum allowed clothing ensemble was significantly correlated with the room temperature. An analytical formulation of this proportion was proposed based on probit analysis. Although within-day changes of clothing also significantly correlated with room temperature, the amplitude of this pattern was much smaller than day-to-day variations.

Further observations were later carried out by Humphreys (1977), but this time with a focus on clothing outdoors. In this, a logistic function of air temperature for the probability of wearing a light clothing ensemble was proposed. It was found that age and sex were not determinant factors, that humidity was not an important variable, and that sunshine had a marginal effect.

Integrating and comparing the observations from these surveys, Humphreys (1979) proposed to model clothing level through an exponentially-weighted moving average of the room temperature. The regression parameters governing the influence of temperature and the exponential decay of the running mean were found not to significantly differ among the datasets.

Measurements performed by Nicol et al. $(1996,1999)$ in offices in Pakistan showed, using linear regression, that the number of items of clothing worn could be associated with both indoor $\left(R^{2}=0.653\right)$ and outdoor $\left(R^{2}=0.666\right)$ temperature; likewise with the comfort vote $\left(R^{2}=0.538\right)$, suggesting that subjects adapt their clothing as a function of perceived thermal comfort. Outside the interval $20-30^{\circ} \mathrm{C}$, clothing insulation was found to remain constant. By way of explanation, Nicol et al. $(1996,1999)$ proposed that clothing removal stops when the limits of acceptable clothing in office environments have been reached. We also suggest that heating systems tend to be used in preference to further clothing additions so that temperatures below $20^{\circ} \mathrm{C}$ are seldom encountered.

Observations of clothing changes performed by Newsham (1997) and Newsham and Tiller (1997), using a computerbased questionnaire, revealed that $15 \%$ of subjects had modified their clothing in the hour prior to the appearance of 
the questionnaire. Approximately twice more clothing removals were recorded than clothing additions.

In a detailed field survey of clothing and activity of 144 subjects, Rowe (2001) observed a diurnal change in clothing insulation value for $38 \%$ of participants, based on detailed indications of garments worn by respondents.

De Dear and Brager (1998) observed that there was a significant correlation $\left(R^{2}=0.25\right)$ between the mean clothing level and indoor temperature, but they found an even stronger correlation with daily mean outdoor effective temperature $\left(R^{2}=0.49\right)$. Morgan and De Dear (2003) subsequently summarised these findings, together with a comprehensive literature review completed by additional research. They underline previous experimental evidence that clothing levels worn indoors are affected by gender (women tend to wear less in summer, but more than males in winter), context, corporate dress codes, indoor climate variability and outdoor weather. They go on to present a model to predict the mean daily clothing value as a linear function of the previous mean daily outdoor temperature and the predicted maximum temperature for the current day.

In summary, the use of clothing in offices is well documented by several field studies of variable size and detail. However, improvements are desirable for the following reasons:

- There is a recurrent problem of measurement reliability for clothing level. Often, a computer-based electronic questionnaire is used; but self-reported insulation levels are approximate with this method.

- The significant variations due to different dress codes in different office environments are appreciated, but not well understood. Furthermore, there is no available research relating to residential environments.

- The influence of indoor or outdoor temperature is well documented. However, data are often analysed with sub-optimal methods such as linear regression on mean clothing values, with a poor quality of fit, which undermines the utility of the models. A comprehensive and validated modelling approach therefore remains elusive.

In this paper, we focus on the latter two issues: we develop a model for the prediction of clothing choices based on rigorously selected predictors, whose formulation should be directly generalisable, given the requisite data, to particular dress codes.

Results of survey and development of model

General patterns A preliminary observation from our dataset is that occupants rarely adjusted their clothing level during the working day; they mostly choose a definite set of garments at the start of the day (or adjust their clothing level upon arrival) which they do not subsequently adjust prior to departure. Among the surveyed periods, lowering clothing level was mentioned for only $1.9 \%$ of the preceding hours, while this fraction falls to $0.2 \%$ for clothing additions (Table 2). However, only relatively coarse categories of clothing were incorporated into our questionnaire, so that minor clothing adjustments may have been neglected.

From the above observations, it seems necessary to distinguish between adaptation of clothing level between days and the occurrence of such adaptations during the day. By this, we mean that occupants may choose their attire at the beginning of the day as a predictive strategy, based on historic experience (e.g. it was warm yesterday and I expect it to be warmer still today, therefore I will reduce my clothing level today) and/or they may wear several layers of clothing and remove these layers as a function of their thermal sensation (e.g. it is cool at the moment, but I expect it to be warm this afternoon, therefore I will provide myself with the possibility to reduce my clothing level during the day). Our preliminary observations indicate that this latter opportunity is seldom exercised (or that it is mostly exercised at the time of arrival) which tends to support the approach adopted by Morgan and De Dear (2003) to predict a static mean daily clothing level. By way of illustration, Fig. 3 (left) shows that seasonal variations have a large amplitude compared to intra-day variations (Fig. 3, right).

These observed proportions of clothing adaptation should, however, be interpreted with caution, since it is possible that small adjustments of clothing level (such as shortening the sleeves or opening the collar of their shirts) are also performed by occupants, whether consciously or not. There may thus exist small but nevertheless significant variations of clothing level of the order of $0.1 \mathrm{clo}$, which may well occur more frequently than the relatively large adjustments that we have observed.

Predicting daily clothing level To facilitate comparisons with previous research, we perform linear regression between observed clothing levels and thermal stimuli. Among outdoor temperature and its derivatives, daily mean outdoor temperature throughout the preceding $24 \mathrm{~h}\left(\theta_{\text {out,dm }}\right)$ produces the best model, far better than indoor temperature $\left(\theta_{\text {in }}\right)$ :

$\mathrm{I}_{\mathrm{cl}}=0.929-0.0184 \theta_{\text {out }, \mathrm{dm}}\left({ }^{\circ} \mathrm{C}\right),\left(\mathrm{R}^{2}=0.405\right)$

$\mathrm{I}_{\mathrm{cl}}=1.719-0.0445 \theta_{\text {in }}\left({ }^{\circ} \mathrm{C}\right)\left(\mathrm{R}^{2}=0.137\right)$

Furthermore, a model including together $\theta_{\text {out,dm }}$ and $\theta_{\text {in }}$ does not yield any significant improvement based on analysis of variance $(F=6.05, p=0.014)$ and the fact that the $R^{2}$ remains almost unchanged at 0.406 . Despite the fact 
Fig. 3 Temporal distribution of observed clothing levels (left monthly evolution, right hourly evolution)
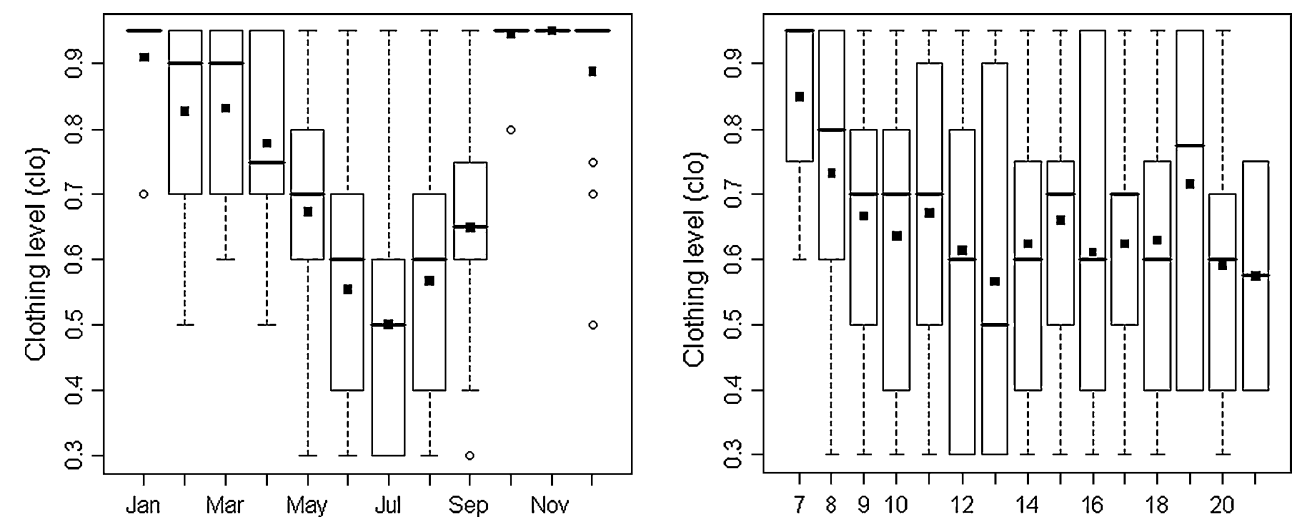

that mean outdoor temperature is a more effective predictor, it is evident from Fig. 4 that this model is associated with considerable dispersion, which suggests the need for a more informative model formulation, i.e. explicitly modelling clothing level distributions rather than considering deceptive unique values (such as mean clothing levels).

Although clothing level is a continuous variable, our questionnaire was designed to only collect approximate values, corresponding to the proposed list of items displayed in Table 1. This was the result of a deliberate compromise between our desire for detailed responses and for numerous repeated responses, so that we did not wish to fatigue our respondents with lengthy lists. Therefore, this questionnaire recorded clothing level as a categorical variable whose ordered discrete levels may be seen as defined by cutpoints applied on an underlying (not measured) continuous variable. An ordinal logistic model is the appropriate tool to use with such data, where the probability for the clothing level $I_{c l}$ to be superior to a given threshold value $I_{j}$ is expressed as a logistic distribution:

$\mathrm{p}\left(\mathrm{I}_{\mathrm{cl}} \geq \mathrm{I}_{\mathrm{j}}\right)=\exp \left(\mathrm{a}_{\mathrm{j}}+\mathrm{b} \theta\right) /\left(1+\exp \left(\mathrm{a}_{\mathrm{j}}+\mathrm{b} \theta\right)\right)$, where $\theta$ is selected as being the most relevant available thermal variable, such as $\theta_{\text {in }}, \theta_{\text {out }}$ or some time-moving average or combination of them. As a candidate variable, we also define the exponentially weighted running mean of the daily mean air temperature as:

$$
\begin{aligned}
\theta_{\mathrm{rm}, \alpha} & =(1-\alpha) \theta_{\mathrm{od}-1}+\alpha \theta_{\mathrm{rm}-1} \\
& =(1-\alpha)\left(\theta_{\mathrm{od}-1}+\alpha \theta_{\mathrm{od}-2}+\alpha^{2} \theta_{\mathrm{od}-3}+\ldots\right),
\end{aligned}
$$

with $\theta_{\text {od-i }}$ being the daily mean temperature $i$ days before and $\alpha$ a constant, usually set to $\alpha=0.8$ in thermal comfort studies.

We fit ordinal logistic models for each of these predictors (Table 3), and observe that the daily mean temperature $\theta_{\text {out,dm }}$ (as opposed to $\theta_{\text {out,rm, }, .8}$ ) is once again the variable with strongest association (Fig. 5 , top left), $\theta_{\text {out, }}$ $\mathrm{rm}, 0.8$ and the instantaneous temperature $\theta_{\text {out }}$ (Fig. 5, bottom left) are the next best predictors. $\theta_{\text {in }}$ performs relatively poorly (Fig. 5, bottom right). In general, the considered averages on $\theta_{\text {out }}$ offer good performance, except for the monthly mean $\theta_{\text {out,mm. }}$.
Fig. 4 Observed (points) and fitted (black line) clothing levels versus daily mean outdoor (left) and indoor (right) temperature. For each discrete clothing level value, the corresponding mean temperature is displayed as a solid square
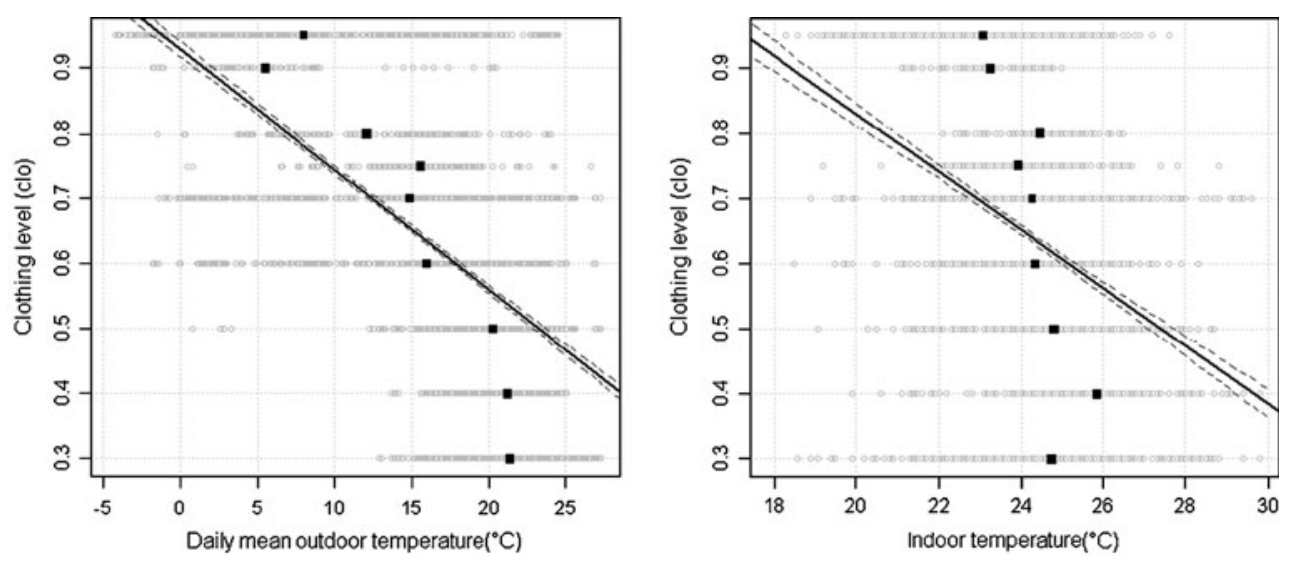
Table 3 Goodness-of-fit estimators for logistic models including one or several variables: likelihood ratio statistic, area under the ROC curve, Somer's $D_{\mathrm{xy}}$, Goodman and Kruskal's $\Gamma$, Kendall's $\tau_{\mathrm{a}}$, Nagelkerke's $R^{2}$ and Brier score (see Haldi and Robinson 2009 for further discussion of these goodness-of-fit estimators)

\begin{tabular}{llllllr}
\hline Variable & LR & AUC & $\mathrm{D}_{\mathrm{xy}}$ & $\Gamma$ & $\tau_{\mathrm{a}}$ & $R_{\mathrm{N}}{ }^{2}$ \\
\hline$\theta_{\text {out }, \mathrm{dm}}$ & $1,763.9$ & 0.760 & 0.520 & 0.523 & 0.444 & 0.400 \\
$\theta_{\text {out,rm, } 0.8}$ & $1,687.4$ & 0.752 & 0.504 & 0.508 & 0.430 & 0.387 \\
$\theta_{\text {out }}$ & $1,677.8$ & 0.748 & 0.497 & 0.499 & 0.424 & 0.386 \\
$\theta_{\text {out,mm }}$ & $1,485.1$ & 0.731 & 0.461 & 0.466 & 0.394 & 0.355 \\
$\theta_{\text {in }}$ & 540.0 & 0.649 & 0.298 & 0.303 & 0.255 & 0.145 \\
$\theta_{\text {out,dm, }} \theta_{\text {in }}$ & $1,767.5$ & 0.757 & 0.514 & 0.515 & 0.439 & 0.104 \\
\hline
\end{tabular}

We have also attempted to add $\theta_{\text {in }}$ as a second variable, but once again its inclusion is not significant according to the likelihood ratio test $(G=3.52, p=0.06)$; it also offers no clear goodness-of-fit improvement (Table 3, last row). Further- more other variables perform no better. We keep thus as a final model for clothing choice Equation 3 with $\theta=\theta_{\text {out,dm, }}$ where

$\mathbf{I}=(0.4,0.5, \ldots, 0.9,0.95)(\mathrm{clo}), \mathrm{b}=-0.1946 \pm 0.0049$

$\mathbf{a}=(5.40 \pm 0.11,4.68 \pm 0.10,4.04 \pm 0.10,3.14 \pm 0.09,2.07 \pm 0.08,1.69 \pm 0.08,1.35 \pm 0.08,1.17 \pm 0.07)$

Finally, we check the ordinality assumption by plotting the means of $\theta_{\text {out,dm }}$ versus the levels of $\mathrm{I}_{\mathrm{cl}}$ (Fig. 5, top right), together with the expected value of $\theta_{\text {out,dm }}$ for each level of $\mathrm{I}_{\mathrm{cl}}$ (shown as a dotted line) under the proportional
Fig. 5 Ordinal logistic regression on clothing level. Observed and fitted distributions of clothing levels with respect to daily mean outdoor (top left) outdoor (bottom left) and indoor temperature (bottom right). Observed prevalence of clothing levels may be read along a vertical line for any chosen temperature. We also verify the proportional odds assumption with daily mean outdoor temperature as a variable (top right)
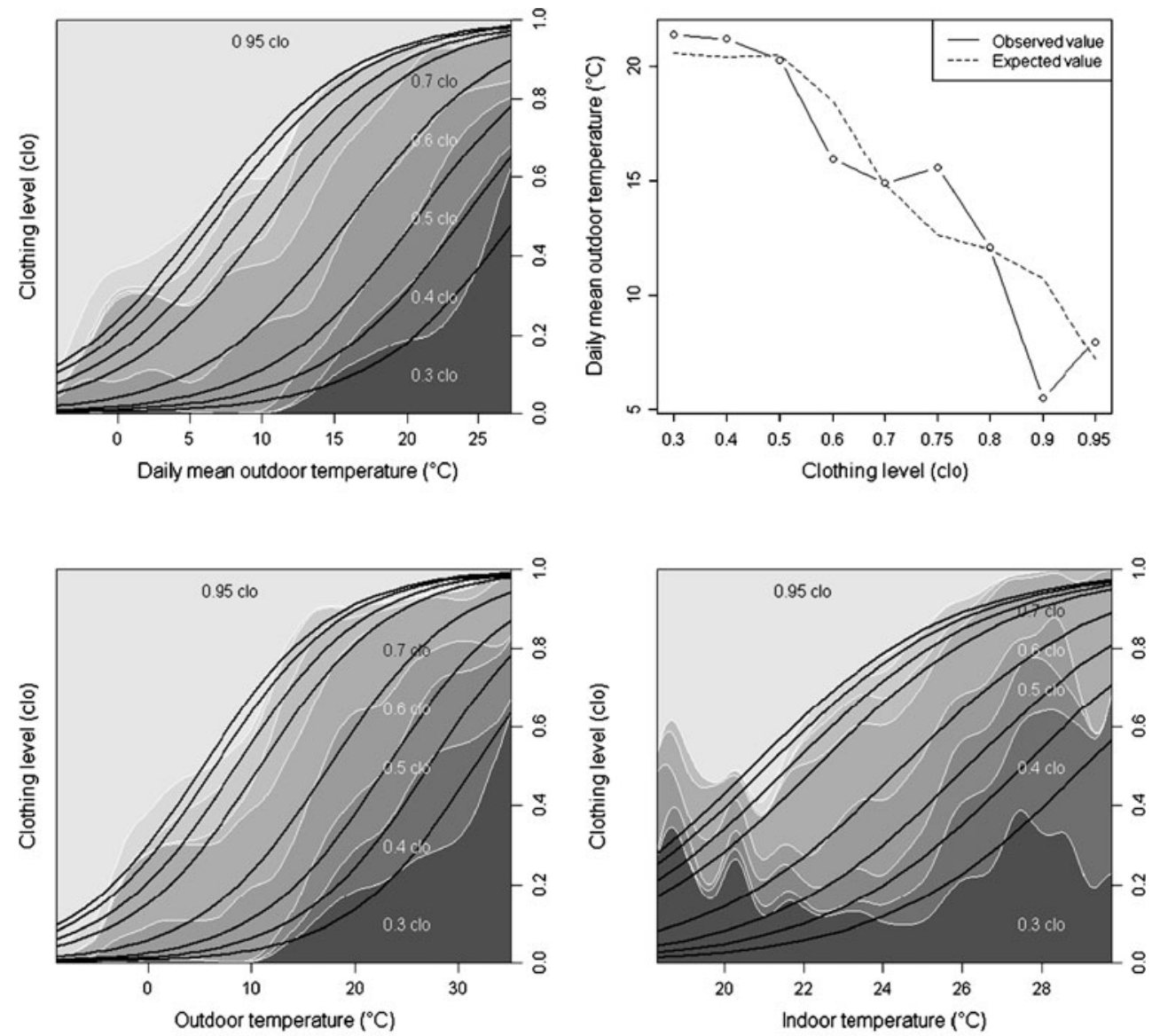
odds assumption. The low observed discrepancy does not show any evidence of violation of the ordinality assumption in this situation.

This model formulation is more informative than ordinary linear regression: it explicitly gives as a result the distribution of predicted clothing levels rather than a mere mean value (which is easily deduced if necessary) and correctly treats the surveyed discrete clothing levels, this being encompassed in a single mathematical expression.

Predicting clothing changes The very small number of observed transitions in clothing level (57 clothing removals and 5 additions) is problematic for the detection of trends in clothing adaptation. We attempted to fit binary logistic models to infer action probabilities and found that there is no significant contribution of any available predictor to explain the variation of the very small observed probabilities.

The rare changes in clothing may be explained by the high degree of general satisfaction with the thermal conditions in the building, or by the availability of other controls to adapt the environment. Indeed, previous studies of occupants' behaviour in the same building have shown that actions on windows (2.40 opening actions per day; Haldi and Robinson 2009) and shading devices (2.02 lowering actions and 1.73 raising actions per day; Haldi and Robinson 2010a) are more frequent than clothing adjustments. Another possible explanation may lie in errors in judgement (occupants may have adapted to a cooler than predicted temperature, so that they have erroneously underclothed themselves).

Further measurements with more precise clothing values would thus be helpful to detect minor clothing adjustments, and better understand occupants' adaptation in clothing. We may, however, hypothesise that determinant predictors should include indoor temperature together with the initial clothing level and its ability to be changed by removing or adding a layer of clothing. This suggests the use of transition probabilities between several clothing states defined by the available clothing items, with $p_{\mathrm{ij}}=0$ for levels $I_{c 1, j}$ that cannot be reached with available clothing $\mathrm{I}_{\mathrm{cl}, \mathrm{i}}$.

\section{Validation and predictive accuracy}

We propose in this section to evaluate the predictive accuracy of our ordinal logistic model using two similar methods. In order to perform an unbiased verification of the predictive accuracy of a model, data used for calibration should be separated from those for verification. Our first approach satisfying this requirement is a cross-validation procedure, in which predictions from a model calibrated using a subset of our measurements (called the training set) are tested on the remaining data (called the validation set). Our second approach, generally considered as more robust, is an independent verification procedure, where a fully independent dataset (the SCATs database; Nicol and McCartney, 2001) is used as validation set, in order to test the capacity of the model to predict occupants' chosen clothing levels in other buildings.

For these two methods, we consider the mean absolute error (MAE) and the root of the mean squared error (RMSE) as predictive accuracy indicators, defined as:

$M A E=\frac{1}{n} \sum_{i=1}^{n}\left|I_{\mathrm{cl}, \mathrm{sim}, \mathrm{i}}-I_{\mathrm{cl}, \mathrm{obs}, \mathrm{i}}\right|$,
$R M S E=\sqrt{\frac{1}{n} \sum_{i=1}^{n}\left(I_{\mathrm{cl}, \mathrm{sim}, \mathrm{i}}-I_{\mathrm{cl}, \mathrm{obs}, \mathrm{i}}\right)^{2},}$

where $n$ is the total number of predictions. Furthermore, we also consider the proportion of correctly classifed clothing levels, exact $\left(\mathrm{r}_{\text {hit, }, 0}\right)$ and up to 0.2 clo $\left(\mathrm{r}_{\text {hit, }, 0.2}\right)$. The results from these indicators are displayed in Table 4.

Cross-validation We perform a ten-fold cross-validation, in which $90 \%$ of the data are used for regression while the remaining $10 \%$ are retained for validation. Such a partition is repeated ten times by permuting this data splitting, so each observation is used nine times for calibration and once for validation. This procedure is itself repeated ten times to detect variations in the performance indicators.

The results of Table 4 indicate that on average the mean absolute error MAE in clothing level prediction lies between 0.180 (clo) and 0.186 (clo) and that the model is able to predict clothing level to within 0.2 (clo) for $62-65 \%$ of cases. Furthermore, detailed examinations show that the signed deviations between predictions and observations are symmetric (Fig. 6, left), suggesting that our predictions are not biased. It is, however, evident that the mean absolute error slightly increases with $\theta_{\text {out,dm }}$, due to the relatively large spread in observed clothing levels at higher temperatures (Fig. 6, top right).

By way of comparison, we have also completed this procedure using $\theta_{\text {out,rm, } 0.8}$ as a variable. From this, we observe a (barely) noticeable decrease in the accuracy indicators, suggesting that this variable may also be used without significant loss in precision.

Independent verification Application of the model to predict the outcomes of a totally independent dataset shows that there is a decrese in predictive accuracy, but only to a very limited extent. For the SCATs longitudinal data, the mean absolute error increases by $5.9 \%$ and the root mean squared error stays almost constant (Table 4). Estimates are 
Table 4 Validation indicators: range of mean absolute error, root mean squared error, proportion of correctly classified outcomes among the ten validation replicates

\begin{tabular}{|c|c|c|c|c|}
\hline Variable & MAE & RMSE & $\mathrm{r}_{\text {hit }, 0}$ & $\mathrm{r}_{\text {hit }, 0.2}$ \\
\hline \multicolumn{5}{|c|}{ Cross-validation $(3,924$ obs.) } \\
\hline$\theta_{\text {out,dm }}$ & $0.180-0.186$ & $0.238-0.242$ & $20.7-22.9 \%$ & $61.7-64.5 \%$ \\
\hline$\theta_{\text {out }, \mathrm{rm}}(\alpha=0.8)$ & $0.184-0.189$ & $0.242-0.247$ & $20.5-22.1 \%$ & $61.4-63.2 \%$ \\
\hline \multicolumn{5}{|c|}{ SCATs long. (27,816 obs.) } \\
\hline$\theta_{\text {out }, \mathrm{rm}}(\alpha=0.8)$ & $0.196-0.199$ & $0.243-0.246$ & $2.4-2.9 \%$ & $60.9-61.6 \%$ \\
\hline \multicolumn{5}{|c|}{ SCATs trans. (4,339 obs.) } \\
\hline$\theta_{\text {out }, \mathrm{rm}}(\alpha=0.8)$ & $0.198-0.205$ & $0.250-0.257$ & $0.4-0.9 \%$ & $55.4-57.9 \%$ \\
\hline
\end{tabular}

slightly less precise for the transverse data (increase in MAE of $8.0 \%$ ) but remain in close agreement. These results would be significantly improved upon if some of the high clothing insulations observed in the SCATs datasets (up to $1.62 \mathrm{clo}$ ) were accounted for in our predictions, which are capped at 0.95 (clo). For information, we display observed and fitted clothing levels from the SCATs longitudinal database in Fig. 7 (left); the ordinal logistic model based on $\theta_{\text {out,rm }, 0.8}$ yields:

$\mathbf{I}=(0.3,0.4,0.5,0.6,0.7,0.8,0.9,1.0,1.1)($ clo $)$,

$\mathrm{b}=-0.1525 \pm 0.0018$

$\mathbf{a}=(9.8 \pm 0.2,5.98 \pm 0.05,4.08 \pm 0.04,1.92 \pm 0.03,1.32 \pm 0.03,0.80 \pm 0.03,-0.32 \pm 0.03,-1.01 \pm 0.03,-2.07 \pm 0.05)$

Optimal time lag of clothing choices The structure of the SCATs data does not explicitly provide daily mean temperatures; however, the exponentially weighted running mean of the daily mean air temperature is provided for nine values of the weighting constant $\alpha$ between 0.33 and 0.99 . Fitting ordinal logistic models using sequentially all these variables shows that the goodness-of-fit indicators reach a maximum among the available values at $\alpha=0.7$ for both SCATs datasets (Fig.7, right), at which the model predictions are relatively robust (as compared to $\alpha \geq 0.8$ ). As mentioned by Nicol and Humphreys (2010), this exponential weighting constant decays the weight of past temper- atures with a half-life of $0.69 /(1-\alpha)$ days. With predictive accuracy being relatively similar within the range $0.45 \leq \alpha \leq$ 0.7 , the corresponding half life is between 1.25 and 2.3 days. However, the optimal value of this weighting constant may depend on the local climatic context.

Discussion of survey and model

Determinant predictors Based on our observations, there is evidence that outdoor climate and season in general primarily determine effective clothing levels in office
Fig. 6 Detailed examination of cross-validation results. Distribution of the obtained signed differences between observed and predicted clothing (left). Mean absolute error versus daily mean outdoor temperature, with local weighted polynomial regression (right)
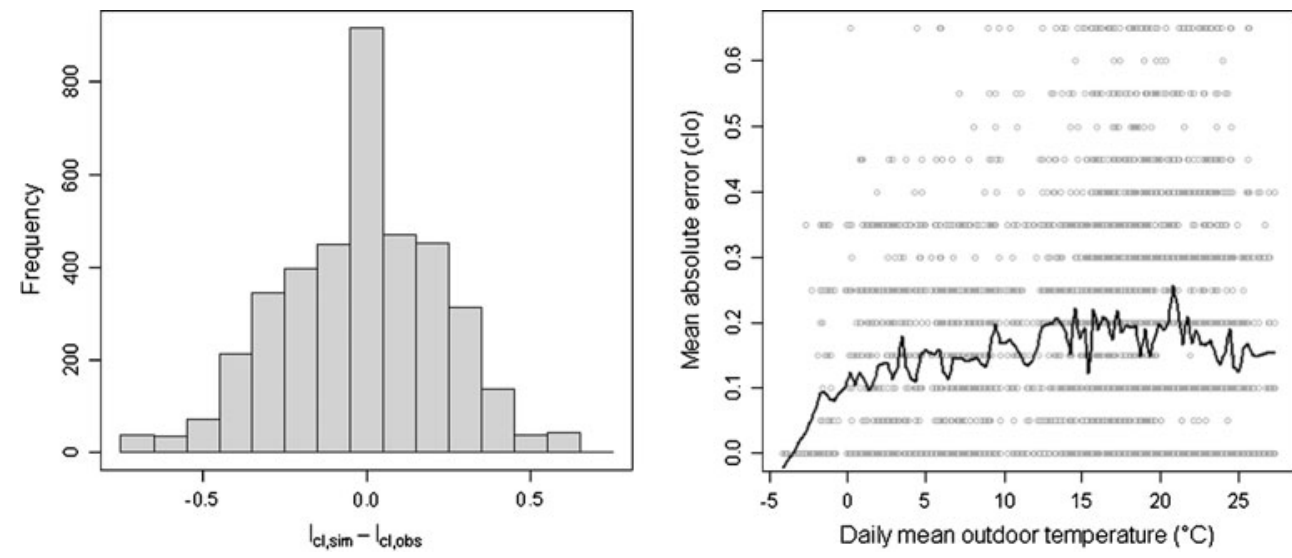
Fig. 7 Observed and fitted clothing levels versus running mean outdoor temperature for the SCATs longitudinal dataset (left). Nagelkerke's $R^{2}$ for ordinal logistic models with different values of the exponential weighting constant of the outdoor temperature (right)

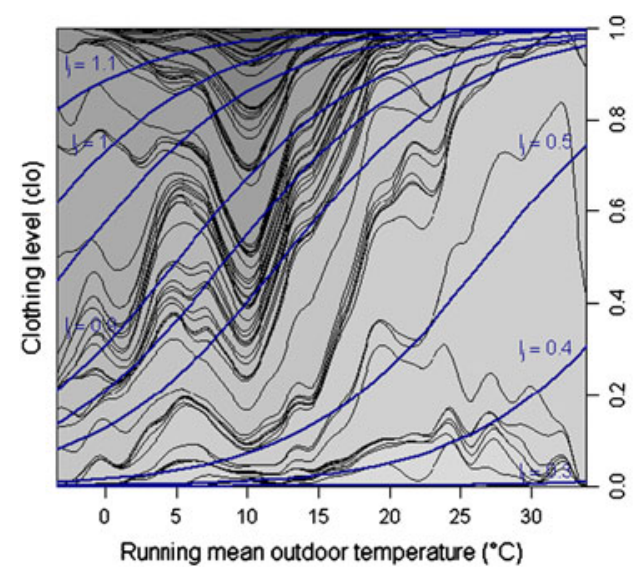

environments. In contrast with other adaptive actions performed by building occupants, such as actions on windows (see Haldi and Robinson, 2009) or blinds (Haldi and Robinson, 2010a), which are consequences of local indoor stimuli, the prevailing outdoor climatic conditions appear to directly influence occupants' clothing decisions. The specific impact of daily mean outdoor temperature is coherent, representing the influence of the recent past on clothing decisions, in conjunction with the fact that changes during the day are rare. It is because of this relative rarity that a model predicting the removal or addition of layers of clothing remains elusive. We may at best offer the seemingly logical conclusion that occupants perform changes (if available) based on local indoor stimuli.

In conclusion, clothing adaptation tends to be more a predictive strategy - the level being set at the beginning of the day, based on prior experience of thermal (especially outdoor) conditions, with opportunities for adaptation during the day being rarely exercised.

Dress codes and specificities In certain office environments, particularly those in which formal attire is favoured, occupants may be rather constrained from adjusting their clothing level.
Occupants of our relatively informal case study building, however, were able to adjust their clothing level in a rather unconstrained way.

A strict dress code implies a suppression of adaptive opportunity (implying a narrower range of acceptable temperatures). This can be modelled using a modified version of Eq. 3 where low clothing levels are removed, and thus merged with the lowest available level. In residential environments on the other hand adaptation may be relatively unconstrained, allowing for yet lower clothing levels if necessary, requiring additional levels in Eq. 3. Figure 8 summarises a possible formulation of these environment-specific distributions.

Proposal for a model Based on these observations, we propose a model formulated as follows:

1. The range of possible clothing levels is determined by the type of environment to simulate.

2. At the start of each day, based on the mean outdoor temperature of the preceding $24 \mathrm{~h}$, a clothing level is randomly drawn from the distribution of Eq. 3; accounting for context-specific constraints.
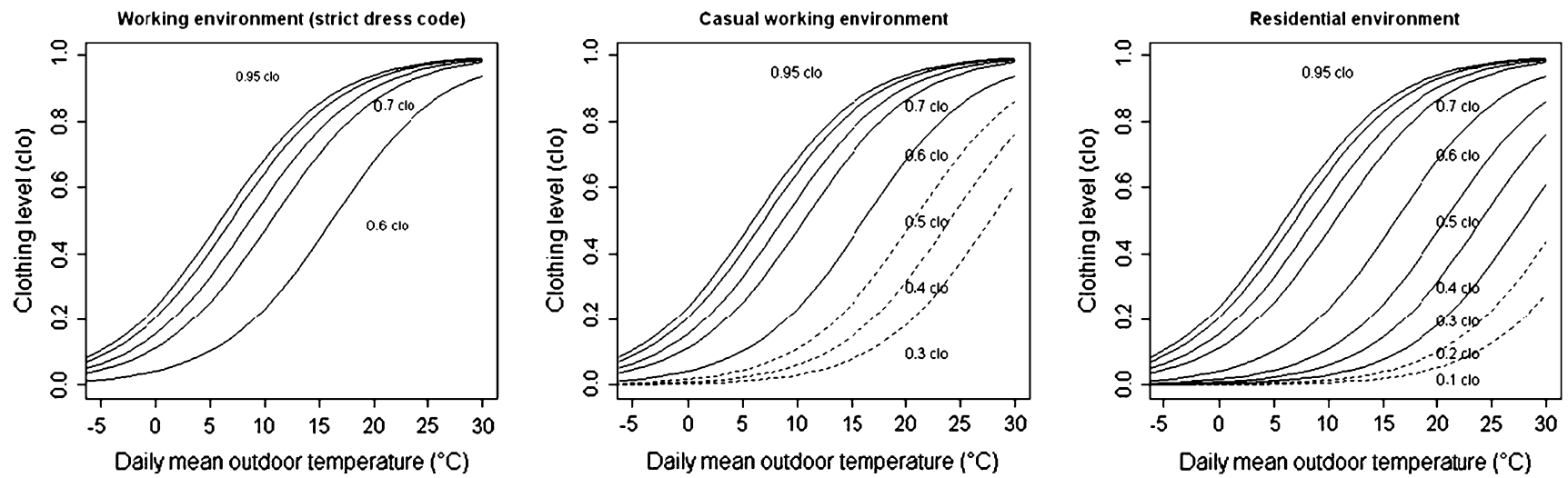

Fig. 8 Hypothetical distributions for clothing in different environments 
3. For each time step where the occupant is present, additions and removals of clothing are predicted using transition probabilities whose explicit form is to be defined.

For such a model, however, dedicated field survey data from residential ${ }^{1}$ and a broader range of non-residential environments would be required. Furthermore, in the absence of data to support the third step above, the relative rarity of this observed form of adaptation would support an intermediate model based on only the first two steps, which models day-today transitions, the most important adaptation mechanism.

\section{Metabolic activity}

Like clothing, metabolic activity is a determinant variable for thermal comfort and is therefore of interest in our research. As with clothing, metabolic activity was selfreported by respondents of our electronic questionnaire, in which a choice from a limited set of metabolic activities (seating, standing, etc.) was made, see Table 1.

State of the art

Research on observed variations of occupants' metabolic activity is very lacunar. A precise measure of this value is also difficult, so that most thermal comfort surveys provide an approximate value calculated from self-reported types of activities. The question is, however, of interest, since this too influences occupants' thermal comfort and so may in principle be used as a strategy to adapt to discomforting thermal stimuli; provided of course that the occupants' tasks permit such adaptations.

In his field survey, Rowe (2001) observed, twice a day, six levels of office activities, using weighting factors for the ongoing duration of this activity and for the consumption of snacks, meals, beverages or cigarettes. Based on collected values ranging between 1.0 and 1.9 met, from $20-27^{\circ} \mathrm{C}$, it was observed that $78 \%$ of respondents reported different activities between morning and afternoon observations. A weak relationship was found between activity rate and indoor operative temperature, but no relationship was found with outdoor temperature.

\footnotetext{
${ }^{1}$ Note that our model is developed from a field survey of the behaviours and preferences of a relatively esoteric group of academics accommodated in a relatively atypical passive solar building (which is warmer than average in winter and may induce correspondingly lower than average clothing levels. The generality of the calibration parameters presented in association with Eq. 3 may thus be placed under scrutiny. We would, however, contest that the form of the model is generalisable (following the above steps and data permitting).
}

Using data from a field survey in Tunisia, Bouden and Ghrab (2005) found that metabolic rate remained nearly constant between 1.2 and 1.3 met, independent of temperature; they were not able to survey occupants after $2 \mathrm{pm}$, when they were absent on siesta: which of course is of itself an adaptive strategy.

\section{Results and discussion}

As with clothing, we attempted to fit an ordinal logistic model for reported categories of metabolic activity levels. But none of the measured variables could explain the very small observed variations in activity levels $(82.7 \%$ of answers indicated sedentary activity, corresponding to $\mathrm{M}=$ 1.2 met). To illustrate the problem, Fig. 9 shows the distribution of activity with respect to $\theta_{\text {out,rm, } 0.8}$ and $\theta_{\text {in }}$.

As noted above, occupants may, in principle, adapt activity levels in response to environmental stimuli, likewise their clothing. But in contrast with residences, workplace activities tend to be dictated by the tasks in hand. In offices, metabolic activity may be particularly constrained, being essentially sedentary (desk-based) in nature. It is, we suggest, due to its constrained nature that we observe no discernible statistical correlation between the adaptation of activity and any kind of environmental stimulus.

It is however possible that, under extreme thermal conditions, occupants vary the intensity of their desk-based activity but our electronic questionnaire was not designed to address this question. There would appear thus to be a need for further field survey data addressing metabolic activities and associated environmental stimuli in less constrained working environments to support the development of a predictive adaptive model.

Outside the working environment, occupants have in principle the freedom to adapt their activities according to their personal preferences (which may or may not be decided in response to environmental stimuli). An obvious and wellknown example is the siesta, which is common to several Mediterranean countries, where activities are slowed down during the peak temperatures of the day (as experienced by Bouden and Ghrab (2005). However, this kind of adaptation is probably a last resort in the range of adaptations to restore thermal comfort. It seems then that there is only marginal interest in modelling variations in metabolic activity as an adaptation response to restore thermal comfort.

\section{Use of drinks}

Taking a cold drink was reported for $18.9 \%$ of hours preceding the questionnaire ( $13.5 \%$ for hot drinks); it is thus a relatively prevalent activity. We have performed logistic regressions for the probability of these events and 
Fig. 9 Observed distribution of activity levels versus exponentially weighted running mean outdoor temperature (left) and indoor temperature (right). Observed prevalence of activity levels may be read along a vertical line for any chosen temperature
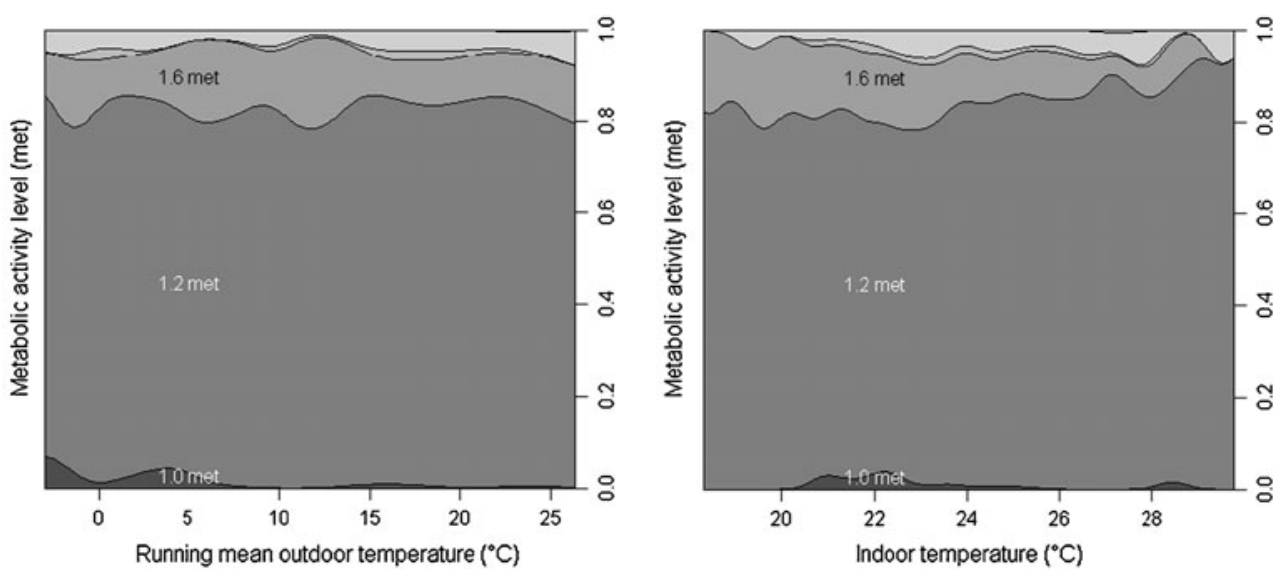

found that indoor temperature is the best predictor for the consumption of cold drinks, while this is the exponentially

weighted running mean of the outdoor temperature $\theta_{\text {out,rm, } 0.8}$ in the case of hot drinks. We obtain then:

$\mathrm{p}_{\mathrm{cd}}\left(\theta_{\text {in }}\right)=\exp \left(\mathrm{a}_{\mathrm{cd}}+\mathrm{b}_{\mathrm{cd}} \theta_{\text {in }}\right) /\left(1+\exp \left(\mathrm{a}_{\mathrm{cd}}+\mathrm{b}_{\mathrm{cd}} \theta_{\text {in }}\right)\right), \quad\left(\mathrm{R}^{2}{ }_{\mathrm{N}}=0.037, \mathrm{AUC}=0.608\right)$

$\mathrm{p}_{\text {hd }}\left(\theta_{\text {out }, \mathrm{rm}, 0.8}\right)=\exp \left(\mathrm{a}_{\text {hd }}+\mathrm{b}_{\text {hd }} \theta_{\text {out }, \mathrm{rm}, 0.8}\right) /\left(1+\exp \left(\mathrm{a}_{\mathrm{hd}}+\mathrm{b}_{\mathrm{hd}} \theta_{\text {out, rm }, 0.8}\right)\right),\left(\mathrm{R}^{2}{ }_{\mathrm{N}}=0.017\right.$, AUC $\left.=0.586\right)$

with $\mathrm{a}_{\mathrm{cd}}=-6.7 \pm 0.6, \mathrm{~b}_{\mathrm{cd}}=0.21 \pm 0.02, \mathrm{a}_{\mathrm{hd}}=-1.32 \pm 0.09, \mathrm{~b}_{\mathrm{hd}}=-0.037 \pm 0.006$.

Cold drink consumption is also modelled with similar accuracy by outdoor temperature, perhaps suggesting the

presence of some form of seasonal adaptation, rather than being influenced solely by internal conditions. The observed

Fig. 10 Observed and fitted probabilities for cold (top) and hot (bottom) drinks to be consumed during the previous hour with $95 \%$ level confidence intervals
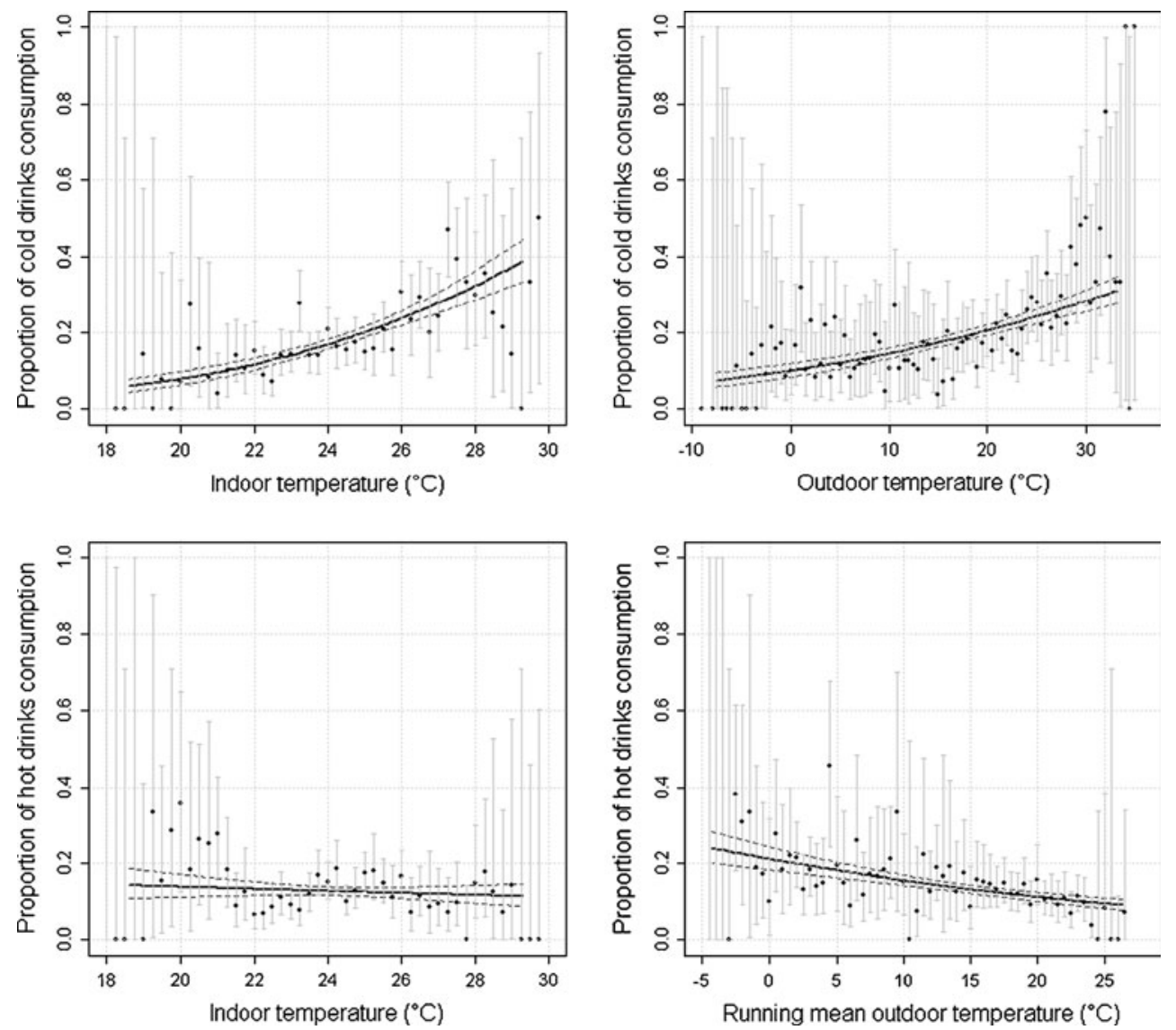
and fitted probabilities are shown in Fig. 10. From this, it seems that building occupants consume cold drinks with greater frequency, relative to a basic minimum (temperature invariant) consumption, when the indoor thermal conditions are warmer; hot drinks are more clearly related with a variable expressing seasonal variations, implying no significant variations during the day.

In conclusion, office occupants seem to adapt their internal heat production in response to thermal stimuli through the use of drinks, which is an unconstrained means of adaptation in a working environment, as opposed to adjustments of metabolic activity.

\section{Conclusions}

Based on careful variable selection, we have presented a predictive model for occupants' daily choices of clothing in naturally ventilated buildings. Its formulation is together:

- Informative: it provides for a probability distribution of occupants' clothing choices;

- Rigorous: the surveyed discrete clothing levels are explicitly modelled;

- Convenient to use and implement: the probability of a given clothing interval is easily deduced, likewise the mean and standard deviation of the clothing level;

- General: application to specific environments with particular dress codes is straightforward, given the corresponding data with which to calibrate the model.

We have also proposed a framework for simulating occupants' clothing adjustments during the day, but are not as yet able to propose a definitive model; this would require considerably more data, ideally from a range of climate types. We have also been unable to develop a meaningful model of variations of metabolic activity in offices. This is because of the relative intransience of this variable in the office context, where activities are relatively constrained. In less constrained environments, metabolic activity may well be a useful adaptive mechanism in response to discomforting thermal stimuli. More data in other types of workplace would be required to investigate this; likewise in respect of adaptations of metabolic activity in residential environments. We have, however, observed a significant impact of thermal conditions on the intake of cold and hot drinks in office environments, which indicates that occupants regulate their internal heat production in this way.

\section{Practical implications}

This study identifies outdoor temperature values in the near past as the key driving variable for building occupants' clothing choices. A detailed statistical analysis of field survey data allow for the formulation of an informative predictive model for the probability distribution of clothing levels in buildings, for application in thermal comfort analysis and building simulation programs. The model is shown to predict clothing insulation values to a 0.2 (clo) precision at a frequency higher than $60 \%$. This research also shows that occupants adapt their metabolic activity through the intake of cold and hot drinks, whose relationship with thermal conditions is demonstrated and can be predicted using binary logistic models.

Acknowledgements Financial support received from the European Commission as part of the CONCERTO II Project HOLISTIC is gratefully acknowledged. We warmly thank the present and former collaborators of our laboratory who contributed to the installation and maintenance of the data acquisition sensors, particularly René Altherr, Antoine Guillemin, David Lindelöf and Laurent Deschamps. We are particularly grateful to Prof. Fergus Nicol for giving us access to the data of the SCATs project and to Revd. Prof. Michael A. Humphreys for his valuable remarks.

\section{References}

Altherr R, Gay J-B (2002) A low environmental impact anidolic façade. Build Environ 37(12):1409-1419

Bouden C, Ghrab N (2005) An adaptive thermal comfort model for the Tunisian context: a field study results. Energy Build 37 (9):952-963

De Dear RJ, Brager GS (1998) Developing an adaptive model of thermal comfort and preference. ASHRAE Trans 104(1A):145167

Haldi F, Robinson D (2009) Interactions with window openings by office occupants. Build Environ 44(12):2378-2395

Haldi F, Robinson D (2010a) Adaptive actions on shading devices in response to local visual stimuli. J Building Perform Simul 3 (2): $135-153$

Haldi F, Robinson D (2010b) On the unification of thermal perception and adaptive actions. Build Environ 45(11):2440-2457

Humphreys, MA (1972) Clothing and thermal comfort of secondary school children in summertime. In: CIB Commission W45 Symposium, Thermal comfort and moderate heat stress.

Humphreys MA (1973) Classroom temperature, clothing and thermal comfort - a study of secondary school children in summertime. J Inst Heat Vent Eng 41:191-202

Humphreys MA (1977) Clothing and the outdoor microclimate in summer. Build Environ 12(3):137-142

Humphreys MA (1979) The influence of season and ambient temperature on human clothing behaviour. In: Fanger PO, Valbjorn O (eds) Indoor Climate. Danish Building Research Institute, Copenhagen, pp 699-713.

ISO (2005) Moderate thermal environments - Determination of the PMV and PPD indices and specification of the conditions for thermal comfort

Morgan C, De Dear RJ (2003) Weather, clothing and thermal adaptation to indoor climate. Clim Res 24(3):267-284

Newsham GR (1997) Clothing as a thermal comfort moderator and the effect on energy consumption. Energy Build 26(3):283291

Newsham GR, Tiller DK (1997) A field study of office thermal comfort using questionnaire software. ASHRAE Trans 103(2):317 
Nicol JF, Humphreys MA (2010) Derivation of the adaptive equations for thermal comfort in free-running buildings in European standard EN15251. Build Environ 45(1):11-17

Nicol JF, McCartney KJ (2001) SCATS: final report. Technical report. Oxford Brookes University, Oxford

Nicol JF, Roaf SC (1996) Pioneering new indoor temperature standards: the Pakistan project. Energy Build 23(3):169-174
Nicol JF, Raja IA, Allaudin A, Jamy GN (1999) Climatic variations in comfortable temperatures: the Pakistan projects. Energy Build 30 (3):261-279

Olesen BW (1985) A new simpler method for estimating the thermal insulation of a clothing ensemble. ASHRAE Trans 91(2B):478-492

Rowe DM (2001) Activity rates and thermal comfort of office occupants in Sydney. J Therm Biol 26(4-5):415-418 